\title{
Research Paper \\ The Effect of Yoga Practice Based on Biorhythms Theory on Balance and Selective Attention of the Elderly Women
}

\author{
${ }^{*}$ Morteza Taheri ${ }^{1} \mathbb{D}$, Khadijeh Irandoust $^{1}$, Ali Seghatoleslami ${ }^{2}$, Mina Rezaei ${ }^{1}$ \\ 1. Department of Physical Education and Sports Science, Faculty of Social Sciences, Imam Khomeini International University, Ghazvin, Iran. \\ 2. Department of Sport Sciences, Faculty of Physical Education, University of Birjand, Birjand, Iran.
}

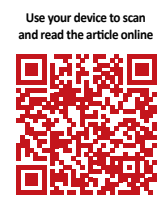

Citration: Taheri M, Irandoust Kh, Seghatoleslami A, Rezaei M. [The Effect of Yoga Practice Based on Biorhythms Theory on Balance and Selective Attention of the Elderly Women (Persian)]. Iranian Journal of Ageing. 2018; 13(3):312323. https://doi.org/10.32598/SIJA.13.3.312

https://doi.org/10.32598/SIJA.13.3.312

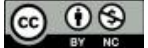

Funding: See Page 321

Received: 3 Mar 2018 Accepted: 16 Jul 2018

Keywords:

Yoga, Balance, Selective attention, Biorhythm

\section{ABSTRACT}

Objectives This study aimed to investigate the effect of yoga based on biorhythm theory on the balance and selective attention in the older women.

Methods \& Materials A total of 35 older women with mean (SD) age of 61.1(2.8) year were randomly assigned into three groups: yoga based on biorhythm $(n=13)$; yoga $(n=13)$; control $(n=11)$. Static and dynamic balance tests and Vienna system test were applied to measure the study variables. COG test was used to measure the selective attention. One-way ANOVA test was used to analyze data followed by post-hoc Tukey test.

Results The results suggested that both motor and cognitive performance, including balance and selective attention were improved significantly ( $\mathrm{P} \leq 0.05)$.

Conclusion Given the physical and mental weakness of elderly women, practicing yoga exercises are highly recommended in the elderly.

\section{Extended Abstract}

\section{Objectives}

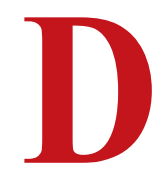

egradation in the physiological, physical and psychological capacities created by the environmental conditions affect the elderly lifestyle resulting in disruption of their daily functioning $[1,2]$. On the other hand, a decrease in physical fitness can disrupt the elderly's motor function and performance and lead to loss of coordination, balance, muscle strength and flexibility, all of which contribute to reducing sensory motor function, instability and increased risk of collapse [3, 4]. Attention to motor and cognitive function of the elderly, as well as their physical condi- tion [5], and considering their specific biological conditions, are the major issues that have always been addressed in the elderly. This research aimed to study the effect of yoga practice based on biorhythms theory on the balance and selective attention of the elderly women.

\section{Methods and Materials}

A total of 35 elderly women with a mean (SD) age of 61.1(2.8) year voluntarily participated in this quasi-experimental study. They were randomly divided into 3 groups: biorhythm yoga $(n=13)$; yoga $(n=13)$; and control $(n=13)$. Inclusion criteria were as follows: older than 60 years, no sleep disturbances (controlled by Pittsburgh sleep quality index), no organized regular activity in the past six months,

\section{* Corresponding Author:}

Morteza Taheri, PhD

Address: Department of Physical Education and Sports Science, Faculty of Social Sciences, Imam Khomeini International University, Ghazvin, Iran. Tel: +98 (912) 4070721

E-mail: m.taheri@soc.ikiu.ac.ir 
Table 1. One-way ANOVA test results about selective attention variables

\begin{tabular}{|c|c|c|c|c|c|c|c|c|c|c|}
\hline \multirow{2}{*}{ Items } & \multicolumn{2}{|c|}{$\begin{array}{c}\text { Biorhythm } \\
\text { Yoga Group (n=13) }\end{array}$} & \multicolumn{2}{|c|}{ Yoga Group (n=13) } & \multicolumn{2}{|c|}{ Control Group (n=13) } & \multirow{2}{*}{ df } & \multirow{2}{*}{$\begin{array}{l}\text { Sum of } \\
\text { Square }\end{array}$} & \multirow{2}{*}{$\mathbf{F}$} & \multirow{2}{*}{ Sig. } \\
\hline & Pretest & Posttest & Pretest & Posttest & Pretest & Posttest & & & & \\
\hline Sum of hits & $21.7 \pm 1.39$ & $25.19 \pm 0.70$ & $2.77 \pm 1.34$ & $2.33 \pm 1.36$ & $22.30 \pm 1.75$ & $22.32 \pm 1.24$ & 2 & 42.62 & 22.34 & $0.001 * * *$ \\
\hline $\begin{array}{l}\text { Sum of } \\
\text { correct } \\
\text { rejections }\end{array}$ & $31.70 \pm 1.08$ & $33.56 \pm 0.7$ & $31.68 \pm 1.33$ & $33.33 \pm 0.65$ & $31.66 \pm 1.30$ & $31.43 \pm 1.07$ & 2 & 17.57 & 8.64 & $0.002^{* * *}$ \\
\hline $\begin{array}{l}\text { Mean time } \\
\text { hits (s) }\end{array}$ & $1.85 \pm 0.041$ & $1.62 \pm 0.03$ & $1.88 \pm 0.05$ & $11.65 \pm 0.11$ & $1.86 \pm 0.05$ & $1.86 \pm 0.04$ & 2 & 0.21 & 50.15 & $0.001 * * *$ \\
\hline
\end{tabular}

acquiring functional independence conditions based on selfdeclaration (the ability to perform routine tasks, 45-minute walking and light exercises), no cognitive disorders such as Alzheimer (self-reported), no depression (controlled by Beck depression inventory), having isocratic diet program (controlled by $\mathrm{N} 4$ software), and body mass index (BMI) between 25-30.

Cognitrone (COG) test within Vienna Test System (VTS) was used to measure selective attention. This test includes the mean time hits, sum hits, and sum correct rejection. The stork test was used to measure static balance, and Timed Up and Go (TUG) test for measuring dynamic balance [4]. Yoga exercises were performed for 8 weeks, 3 sessions per week including Asana, Pranayama and meditation exercises [6].

The first group (biorhythm yoga) exercises were designed based on the biorhythm chart of each individual. To do so, first, based on the date of birth and its entry into the biorhythm software, the physical, emotional, cognitive, and perceptual status of the subjects was extracted, and then the program was designed accordingly. In this group, the 16 days when the quadruple status of the subjects was above $50 \%$, were selected as training days. The t test, 1-way ANOVA and Tukey post hoc test were used at the significant level of 0.05 for data analysis.

\section{Result}

Based on KS test resulting in normally distributed data, the parametric test was used. The results of 1-way ANOVA(Table 1) showed a significant difference in the selective attention variables among three groups $(\mathrm{P} \leq 0.05)$. The mean time hits (Figure 1) indicated that the biorhythm yoga and yoga groups had a significant improvement after the intervention compared to the control group $(\mathrm{P}=0.001$ and $\mathrm{P}=0.005$, re- spectively), and there was no significant difference between these groups $(\mathrm{P}=0.44)$

One-way ANOVA test results showed a significant difference between the static and dynamic balances in subjects after intervention $(\mathrm{P} \leq 0.005)$. According to Tukey post hoc test results about static balance, the biorhythm yoga and yoga groups had a significant improvement after the intervention compared to the control group $(\mathrm{P}=0.023$ and $\mathrm{P}=0.032$, respectively) and there was no significant difference between these groups $(\mathrm{P}=0.65)$. The results of $\mathrm{t}$ test showed that both static and dynamic balances improved significantly in both experimental groups $(\mathrm{P} \leq 0.05)$, while there was no significant difference in the control group.

Tukey post hoc test results about dynamic balance also revealed that the biorhythm yoga and yoga groups had a significant improvement after the intervention compared to the control group $(\mathrm{P}=0.001$ and $\mathrm{P}=0.002$, respectively $)$ and there was no significant difference between these groups $(\mathrm{P}=0.43)$.

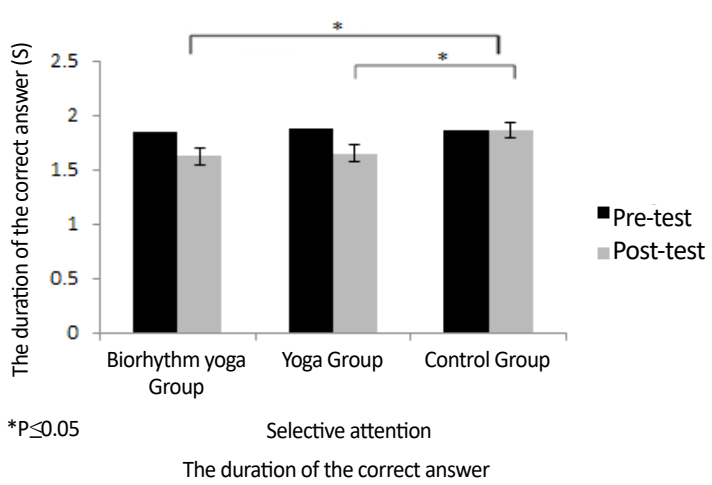

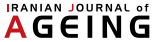

Figure 1. Comparison of mean time hits among three groups before and after intervention 


\section{Conclusion}

Research in the field of executive functions (motor/cognitive factors) in the elderly is limited. Since balancing and improving cognitive performance are very important in old age, evaluation of balancing performance as well as executive performance of the elderly following effective exercise protocols are strongly recommended. Balance and selective attention have the potential to improve with exercises like yoga, which is very important in motor control. Improvement of selective attention and balance can help elderly people in two categories of cognitive and motor abilities, and result in their well-being. However, there is a need for further research on the effectiveness of biorhythms, since it is affected by psycho-biological functions, too.

\section{Ethical Considerations}

\section{Compliance with ethical guidelines}

This study was conducted after obtaining the permission of the Ethics Committee of Imam Khomeini International University (Ethics Code: 17628).

\section{Funding}

This research did not receive any specific grant from funding agencies in the public, commercial, or notfor-profit sectors.

\section{Conflict of interest}

The authors declared no conflict of interest.

\section{Acknowledgements}

Hereby, we appreciate all the people who participated in this research specially Ms. Zahra Dadmehr and Maryam Irandoost who helped us with collecting data. 


\title{
تأثير تمرين يوكًا مبتنى بر نظريه بيوريتم زيستى بر تعادل و توجه انتخابى زنان سالمند
}

\author{
"مرتضى طاهرى' '، خديجه ايراندوست'، على ثقة|لاسلامى'، مينا رضايى' \\ | - كروه تربيت بلنى، دانشكده علوم اجتماعى، دانشكاه بينالمللى امام خمينى، قزوين، ايران.

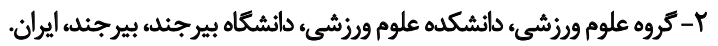

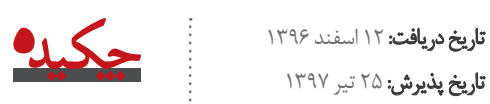

الهداف هدف از اين تحقيق بررسى تأثير تمرين يوكا مبتنى بر نظريه بيوريتم زيستى بر عملكرد تعادلى و توجه انتخابى سالمندان بود.

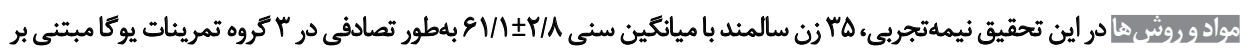

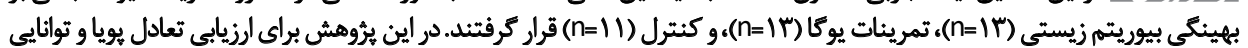

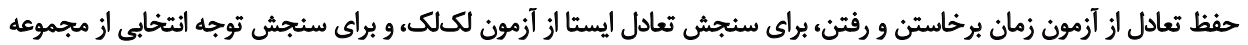

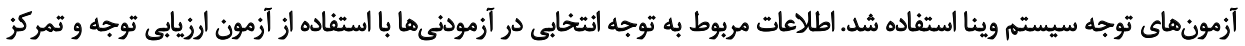

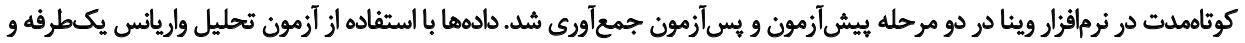

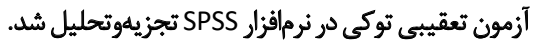

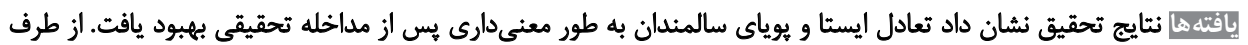

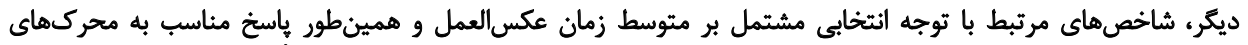

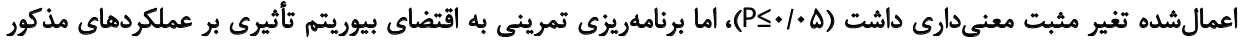
نداشت.

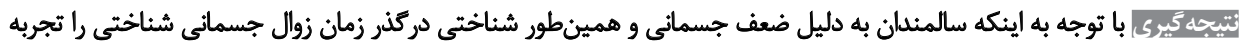

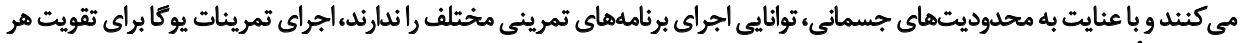
دو بخش تأكيد مي كئشود.

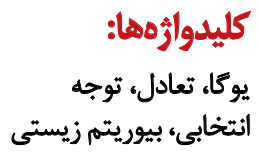

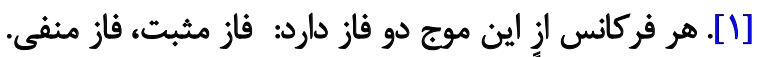

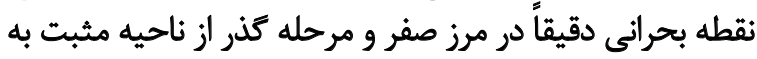

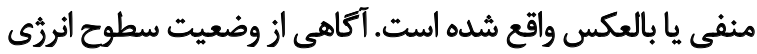

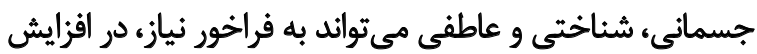

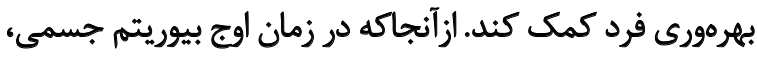

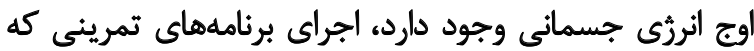

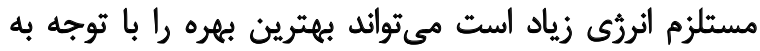

وضعيت جسمانى داشته باشد [1، [1]].

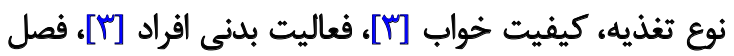

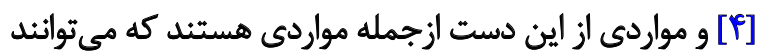

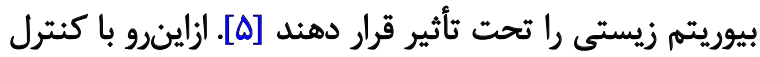

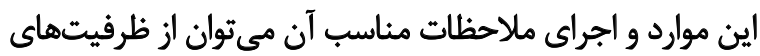

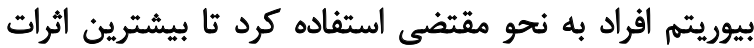

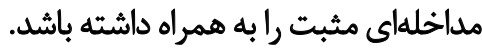

در علوم شناختى يرداختن به ذهن و اثرات آن بر فيزيولوريى و

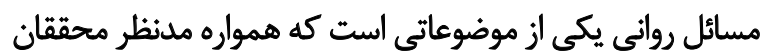

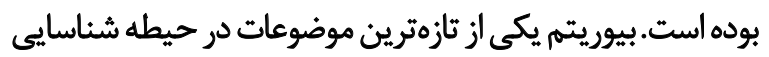

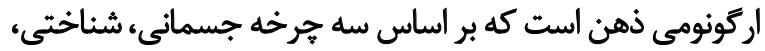

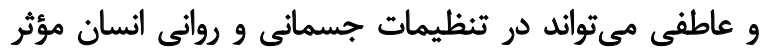

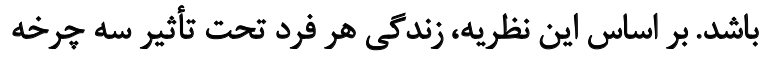

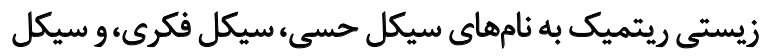

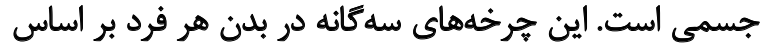

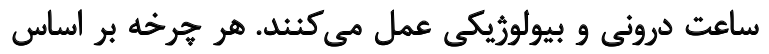

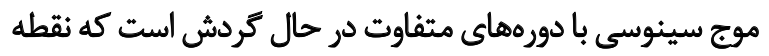
شروع همه آنهاء روز تولد فرد است.

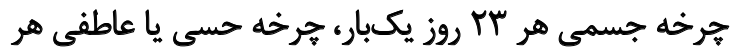

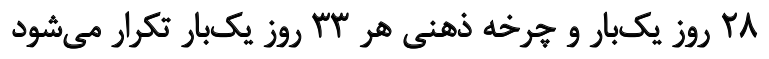

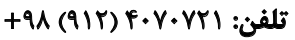
m.taheri@soc.ikiu.ac.ir يست الكترونيكي إن، 


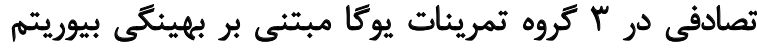

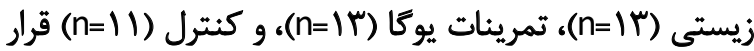

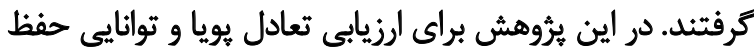

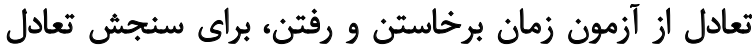

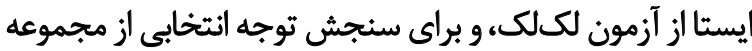

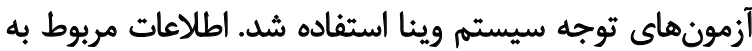

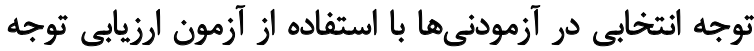

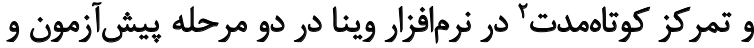

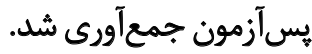

معيارهاى ورود به مطالعه عبارت بودند از: داشتن سن بالاى

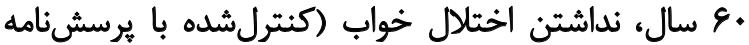

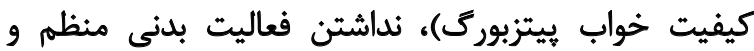

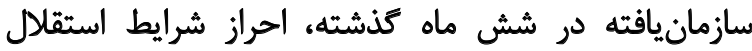

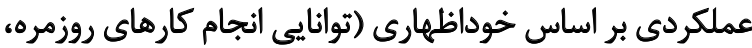

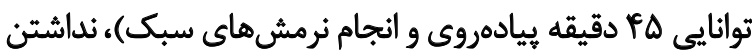

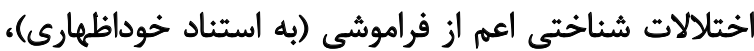

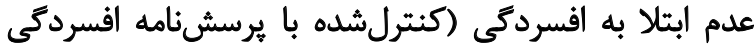

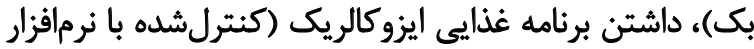

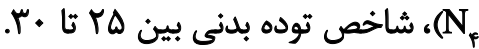

در مرحله بيش آزمون و يسآزمون از آزمون الرزيابى توجه

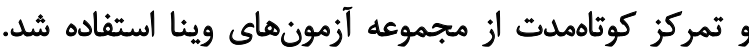

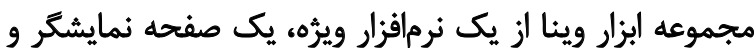

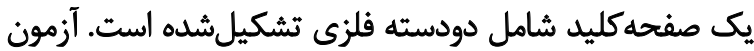

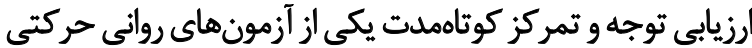

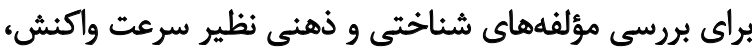

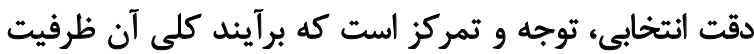

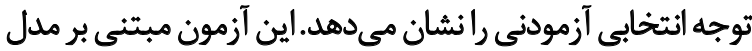
نظرى رولخ (1991) است.

بر اساس اين مدل تمركز از سه جزء انرزئ (ميزان انرئى

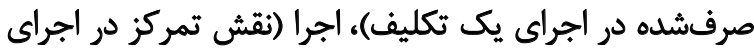

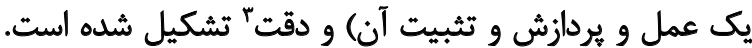

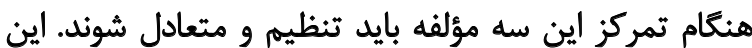

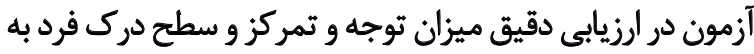

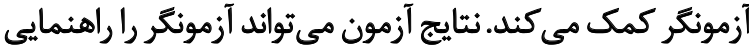

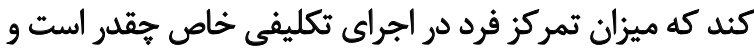

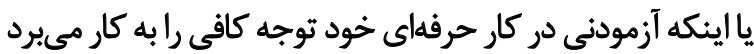

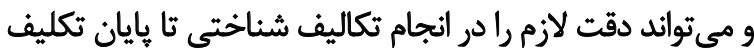

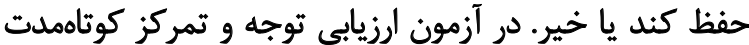

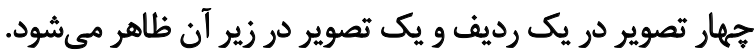

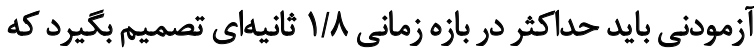

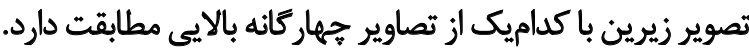

2. Cognitrone (COG)

3. Precision
حيطه كنترل حركتى از موضوعات بسيار مهم و كليدى است

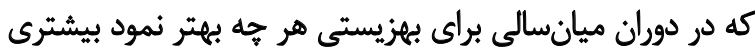

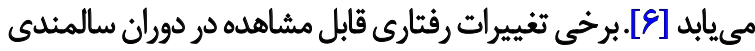

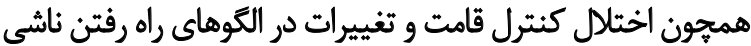

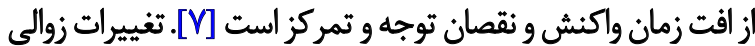

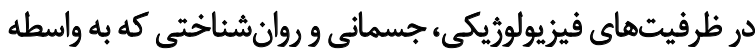

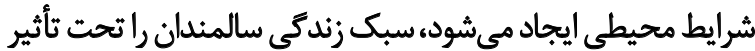
قرار مى دهد و درنهايت عملكرد روزانه سالمند را مختل مى كندي كاهش آمادكى جسمانى مي تواند عملكرد و قابليتهاي حركتى

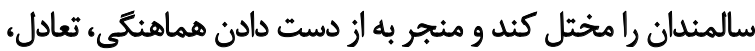

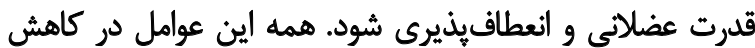

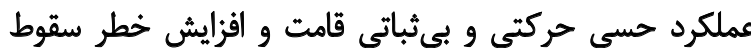

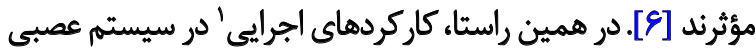

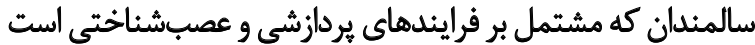

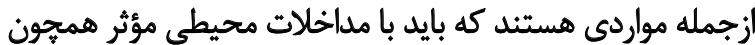

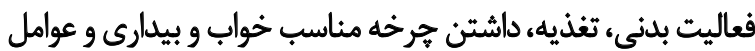
محيطى ديكر مورد توجه قرار گيرند. بيوريتم زيستى با تقسيمات جهار كانه (ريته جسمانى، احساسى،

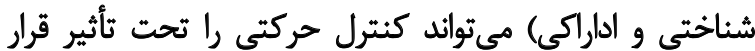

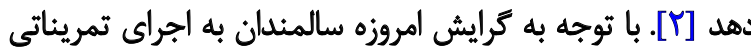

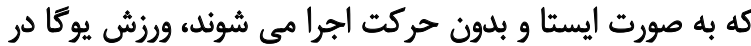

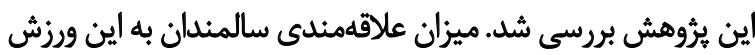

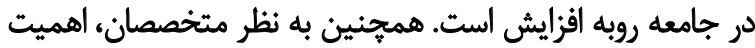

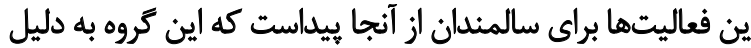

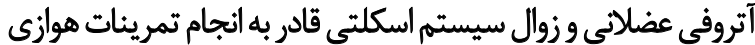
و مقاومتى معمول نيستند و در اين صورت احتمال آسيبديديدى آترئي

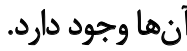

آثار مثبت مداخلات حركتى و تمرينى همجون برنامه ورزش در

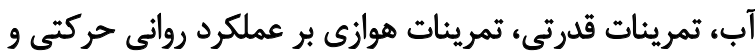

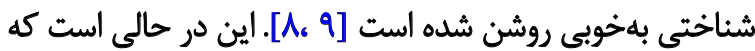

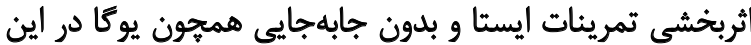

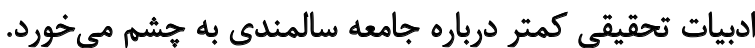

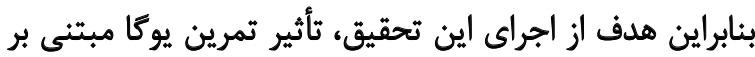

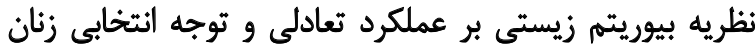

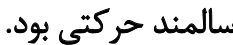

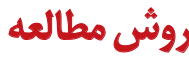

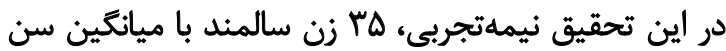

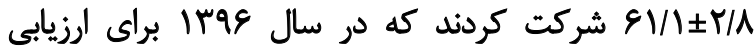

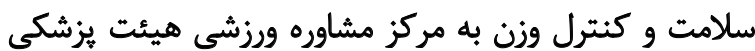

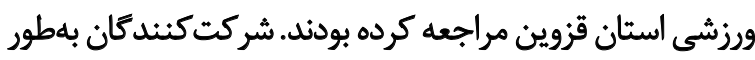


Primaty thythms on 02 Sep 2017

for fateme hodeini
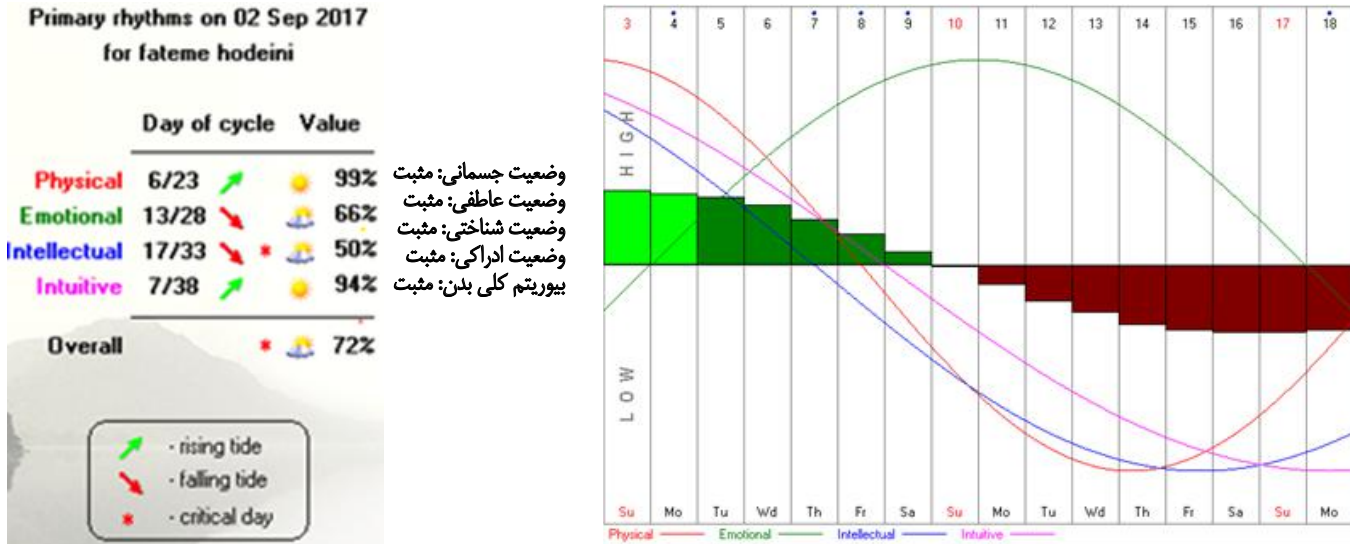

تصوير ا. نمودار بيوريتيهم آزمودني در طول يك بازه زماني (شكل راست) و در يك روز (شكل جي)

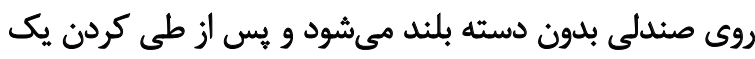

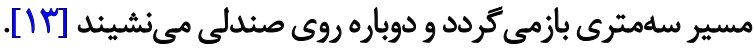

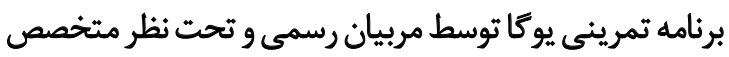

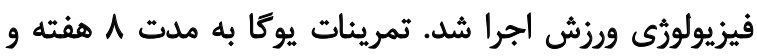
r ب جلسه در هفته انجام شد كه شامل تمرئ تمرينات

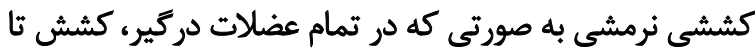

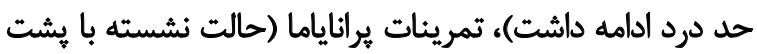

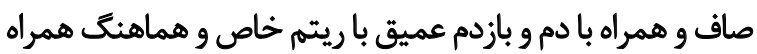

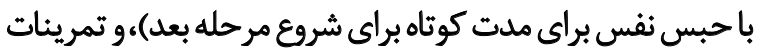

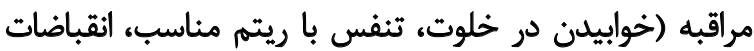

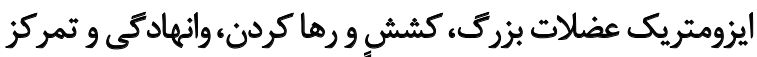

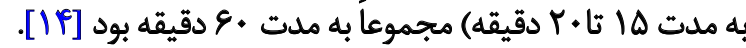
در بازه زمانى دو ماهه مداخلات تمرينى، ابتدا بر اساس تاريخ

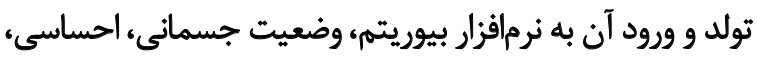

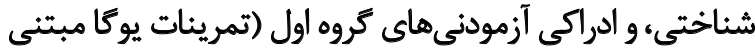

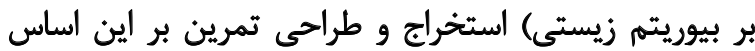

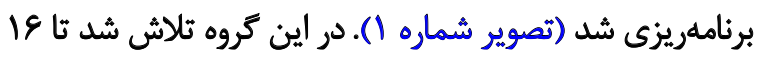

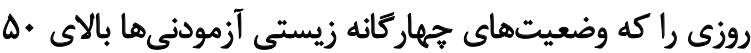
درصد بود، به عنوان روزهاى تمرين طراحى شود. طرح تحقيق در جدول شماره ا قابل مشاهده است. ازآنجاكه

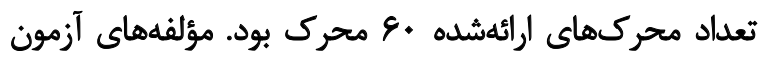

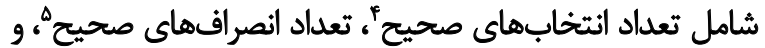
ميانكين زمان انتخاب صحيح "در آزمونها بود [ـ • [1]. از آزمون استورك' براى سنجش تعادل ايستا بر حسب ثانيه

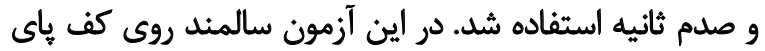

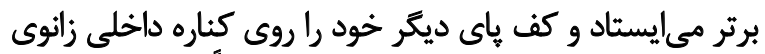

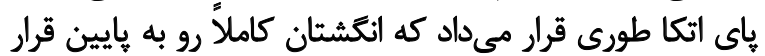

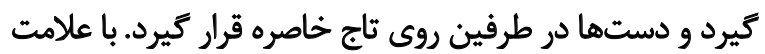

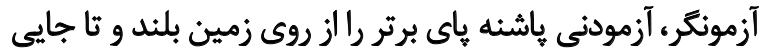

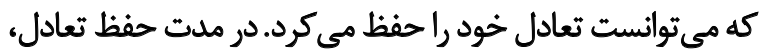

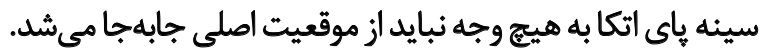

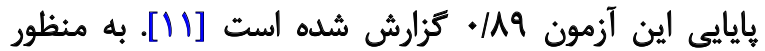

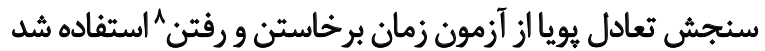

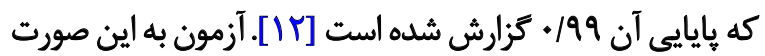

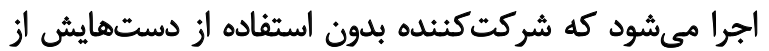

\section{Sum hits}

5. Sum correct rejection

6. Mean time hits

7. Stork test

8. Timed Up and Go (TUG)

جدول ا. طرح تحقيق

\begin{tabular}{|c|c|c|c|c|c|}
\hline \multicolumn{2}{|c|}{ ساعت ج صبح: يس آزمون از خوردن } & \multirow{2}{*}{ 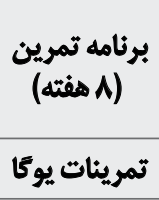 } & \multicolumn{2}{|c|}{ ساعت و صبح: يس أز خوردن صبحانه آيزوكالريكى } & \\
\hline آزمون توجه اثتخابي & آزمون تعادل & & آزمون توجه انتخابى & آزمون تعادل & \\
\hline$\checkmark$ & $\checkmark$ & & $\checkmark$ & $\checkmark$ & يوكا (برينكى بيوريتم) \\
\hline$\checkmark$ & $\checkmark$ & & $\checkmark$ & $\checkmark$ & يوكا \\
\hline$\checkmark$ & $\checkmark$ & & $\checkmark$ & $\checkmark$ & كنترل \\
\hline
\end{tabular}

r 


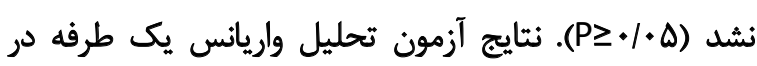

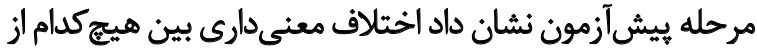

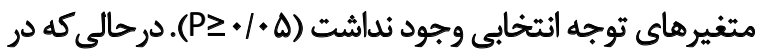

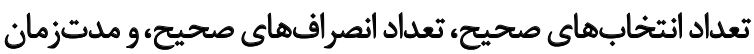
انتخاب صحيح بهبود عملكرد مشهود بود. نتايج آزمون تعقيبى توكى در تصوير شماره r ديده مى مشود.

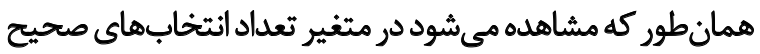

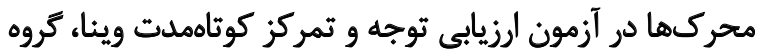

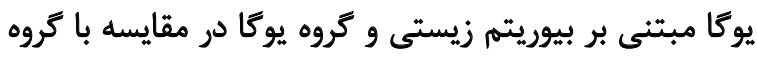

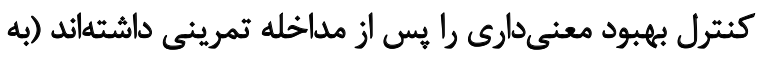

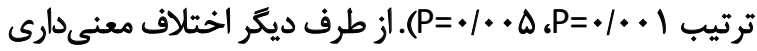

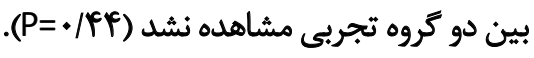

همانطور كه در تصوير شماره ب ديده مىشوده، متغير تعداد

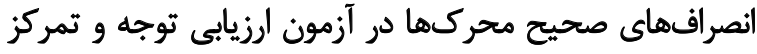

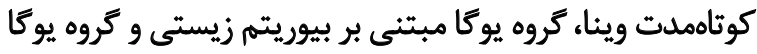

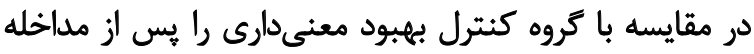

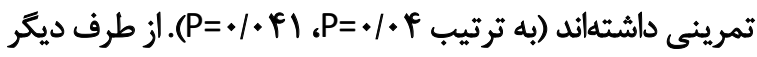

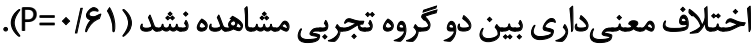
همانطور كه در تصوير شماره f ديده ميشود، متغير مدت
بمامى افراد تحت يوشش كلينيك بودند، برنامه غذايي با توجه

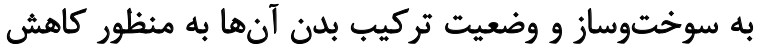

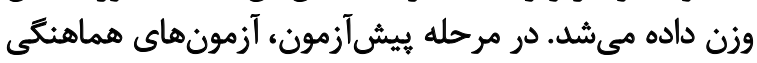

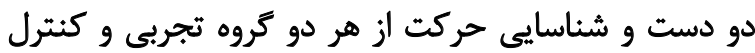

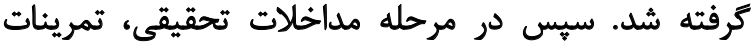

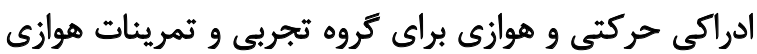

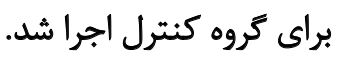

اين مطالعه يس إز كسب مجوز كميتهاخلاق دانشكاه بينالمللى

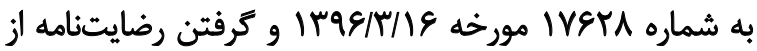

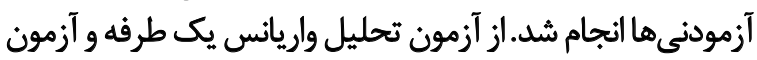

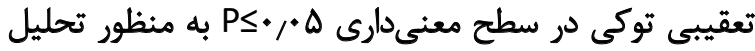

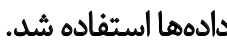

ياقتهها

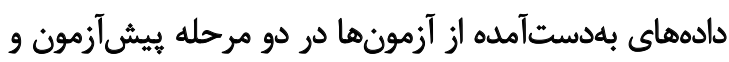

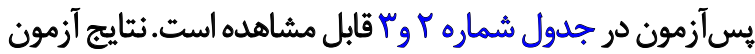

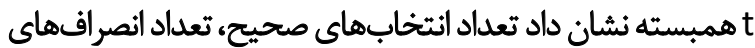

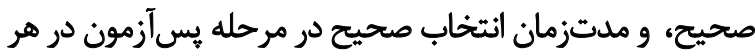

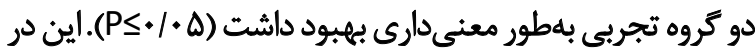

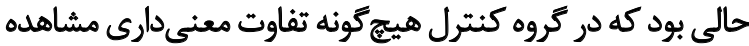

جدول Y. نتايج آزمون تحليل واريائس يكطرفه در خصوص مقايسه متغيرهاى توجه انتخابى در كروهها

\begin{tabular}{|c|c|c|c|c|c|c|c|c|c|c|c|}
\hline \multirow[t]{2}{*}{$\mathbf{P}$} & \multirow[t]{2}{*}{$\mathbf{F}$} & \multirow{2}{*}{ مجذياتئين } & \multirow{2}{*}{ أزوجى } & \multicolumn{2}{|c|}{ كترل (n=IT) } & \multicolumn{2}{|c|}{ تمرين يوكًا (n=) } & \multicolumn{2}{|c|}{ 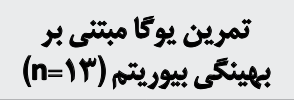 } & \multirow[t]{2}{*}{ كروه/آزمون } & \multirow[b]{2}{*}{ متغير } \\
\hline & & & & هسآزمون & يثيش آزمون & يسآزمون & ييشآزمون & هسآزمون & بيشي آزمون & & \\
\hline $1 . .11^{-00 *}$ & $T r / M r$ & PT/RT & $r$ & $M T / N \pm M / M E$ & $M / / \pm \Psi \cdot / N \Delta$ & $r / \triangle \pm M / T E$ & $r / / \pm W / M F$ & $r \Delta / \cdot \pm 18 V$ & $r V / \backslash \pm V / T q$ & صحيح تثخابهاي & \\
\hline $.0 .+4=* *$ & NAT & IV/OV & r & $r V / \pm N T / \cdot V$ & $r V I \pm E g / \mu$. & $M T / * \pm M / F_{\Delta}$ & $M / / \pm E N M$ & $\pi /+ \pm \Delta S N$ & $r V \backslash \pm V+/ * \Lambda$ & |تصرافهالى تعليح & $\begin{array}{l}\frac{2}{4} \\
\frac{5}{3} \\
\frac{13}{3}\end{array}$ \\
\hline $.1 .01^{n * * *}$ & $\Delta . / 1 \Delta$ & $\cdot|M|$ & $r$ & $V \cdot \pm N \& \mid \cdot f$ & $V \cdot \pm N \mid \cdot \Delta$ & $V \cdot \pm 8 \mathrm{~A} / 11$ & $V \cdot \pm M \cdot \Delta$ & $V / \cdot \pm R / \cdot r$ & $V / \pm N Q / \cdot f$ & ملتخزمان & \\
\hline
\end{tabular}

جدول r. نتّايج آزمون تحليل واريائس يك طرفه در خصوص تعادل يويا

\begin{tabular}{|c|c|c|c|c|c|c|c|c|c|c|}
\hline \multirow[t]{2}{*}{$\mathbf{P}$} & \multirow[t]{2}{*}{$\mathbf{F}$} & \multirow{2}{*}{ مجذورات مينين } & \multirow{2}{*}{ أز أدادى } & \multicolumn{2}{|c|}{ كتترل } & \multicolumn{2}{|c|}{ يوكا } & \multicolumn{2}{|c|}{ تمبينَّي بيوكا مبتنى بر بيتر } & \multirow[t]{2}{*}{ 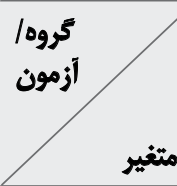 } \\
\hline & & & & يسآزمون & يبشآزمون & يَسآزمون & ييشآزمون & يسآزمون & بيشأزمون & \\
\hline $.1 .010 *$ & $W \cdot G$ & $\Delta(r)$ & $r$ & $N \cdot \pm r \mid M I$ & $N \cdot \pm \mid V A r$ & $V / \cdot \pm r V A F$ & $N \cdot \pm T r / W$ & $q / . \pm q 4 / \Delta r$ & $N \cdot \pm I \sqrt{9} \Delta$ & تعادل يويا (ثانيه) \\
\hline.$|0 .|^{* \ldots *}$ & IV/8. & INAY & $r$ & $|Q| \cdot \pm \mid Q / V$ & $\mid \alpha / 1 \pm \cdot V \cdot \Delta$ & $191 . \pm 999 \Delta$ & $|Q /| \pm \cdot V \mid \cdot q$ & $|V| \cdot \pm \cdot 8 / 8 \Delta$ & $10 / \cdot \pm \cdot 8 / W$ & تعادل إيستا (ثانيه) \\
\hline
\end{tabular}




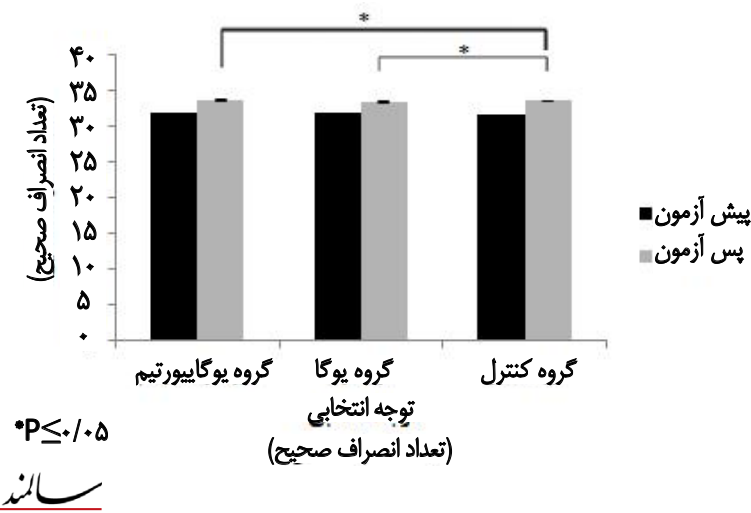

تصوير بّ. توجه انتخابى سالمندان (متغير تعداد انصرافهاى صحيح) ييش و

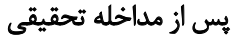

ب

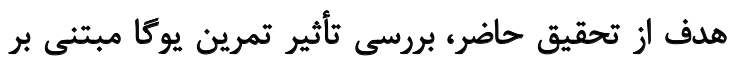

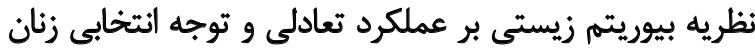

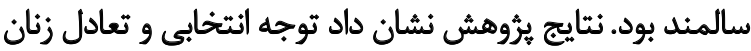

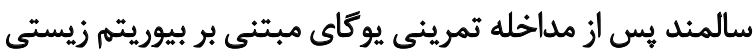

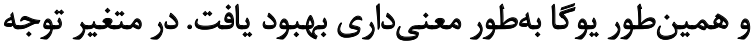

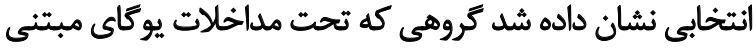

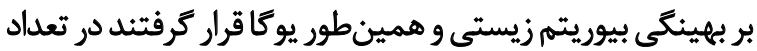

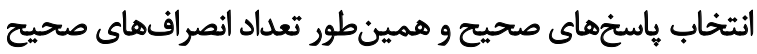

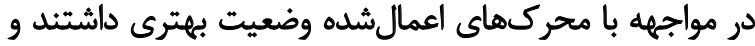

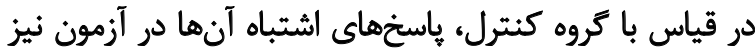

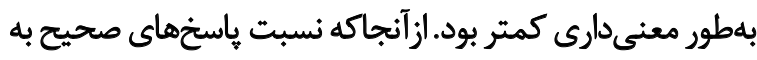

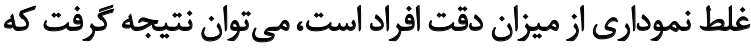

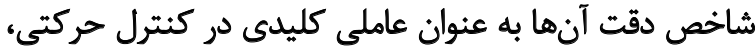

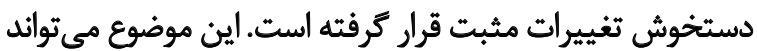

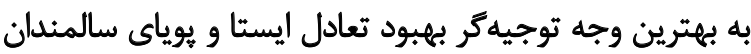

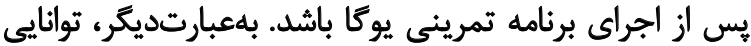

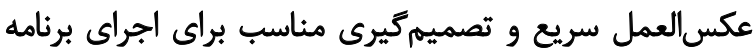
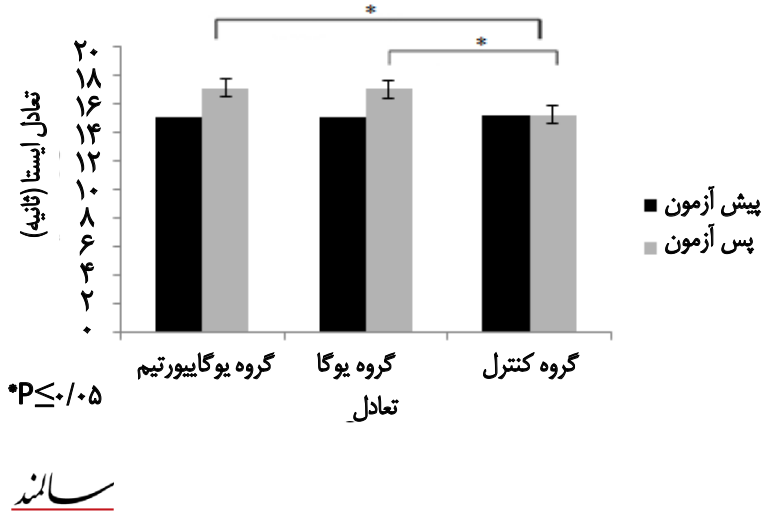

تصوير هـ مقايسه تعادل ايستاى زنان سالمند بيش و يس از مداخله تحقيقي

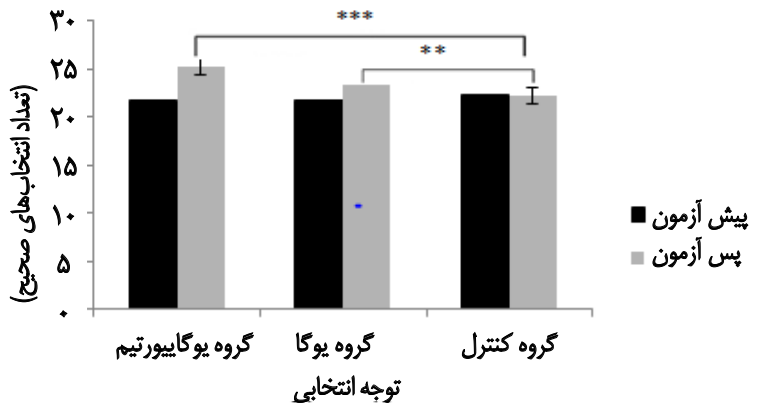

*PS

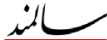

تصوير r. توجه انتخابى سالمئدان (متغير تعداد انتخابهاي صحيح) بيش و

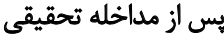

زمان انتخاب باسخ صحيح در آزمون ارزيابى توجه و تمركز

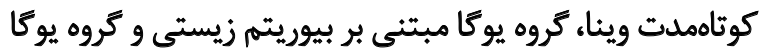

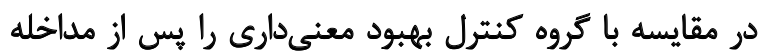

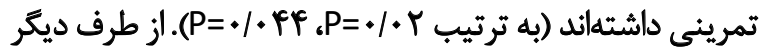

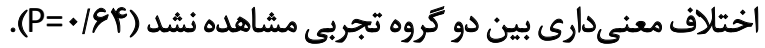

نتايج آزمون توكى در خصوص تفاوت ميان تروهها در تعادل

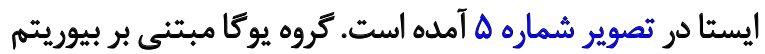

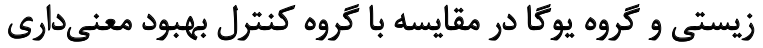

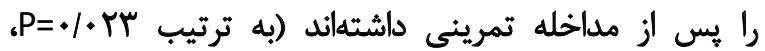

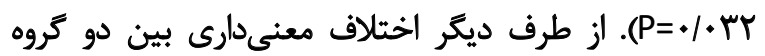

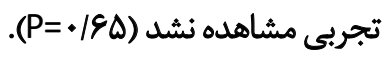

نتايج آزمون توكى در خصوص تفاوت ميان كروهها در تعادل

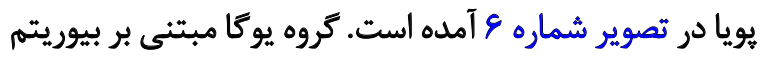

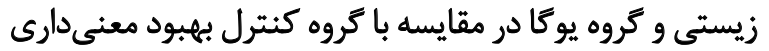

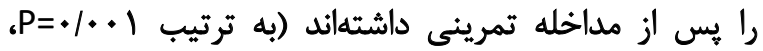

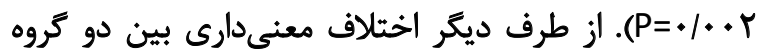

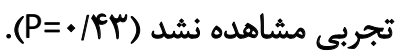

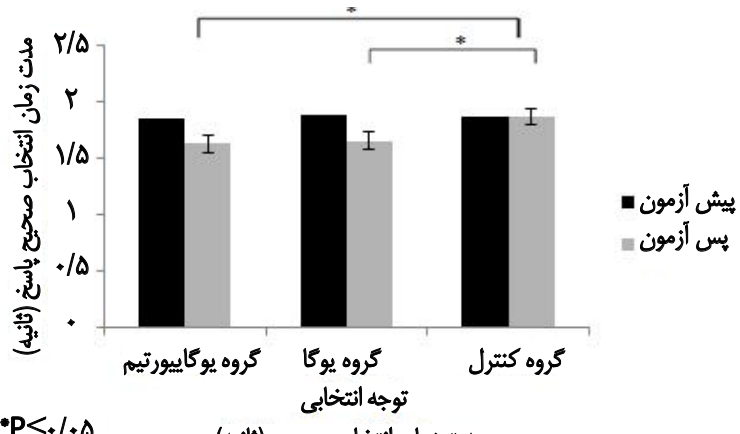

•P مدت زمان الثتخاب صحيح (ثانيه)

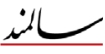

تصوير †. مقايسه توجه انتخابي سالمندان (مدتزمان انتخاب ياسخ صحيح)

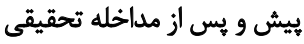


را در سالمئدان به همراه خواهد داشت [1ه]. متوسط زمان عكسالعمل بهعنوان بخش مهمى ازئ عامل توجه

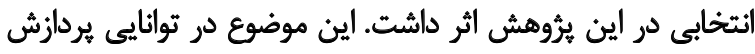

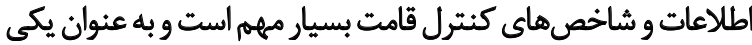

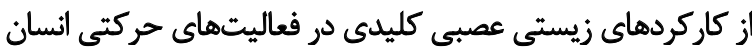

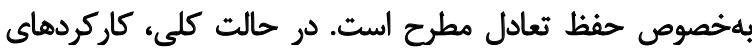

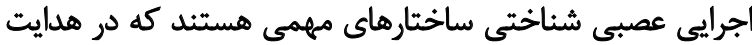

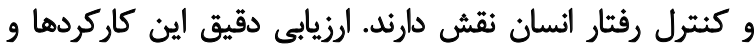

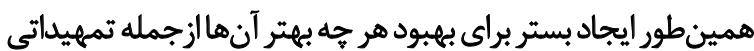

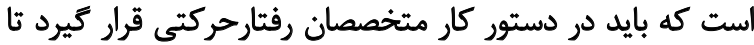

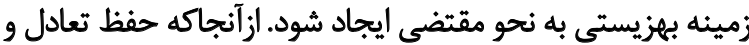

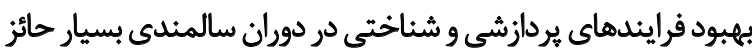

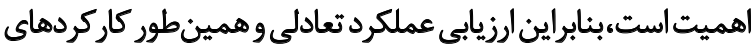

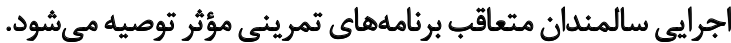

\section{نتيجليَيرى نمايى}

تحقيقات در حوزه كاركردهاى اجرايى سالمندان كه عوامل

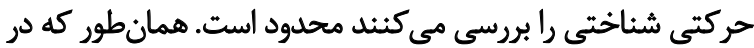

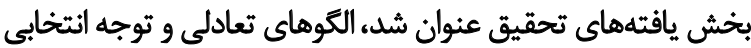

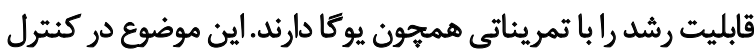

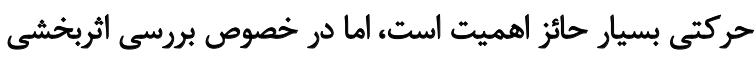
بيوريتم به تحقيقات بيشترى نياز است.

يكى از محدوديتهاي اين مطالعه تعداد نسبتاً اندى آزمودنى هاو

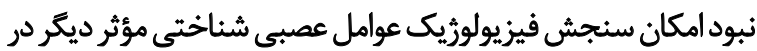

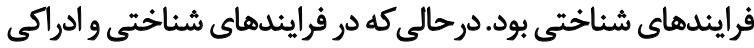

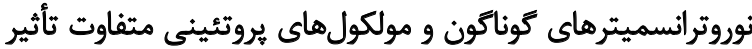

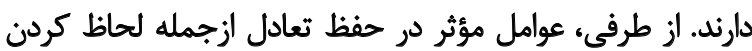

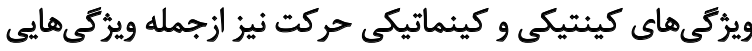
است كه مىتواند به قوت جنين و تحقينياتيكي حركي بيفزايد.

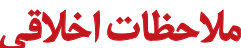

\section{يبيروى از اصول اخلاق يثوهش}

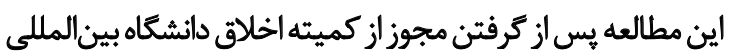

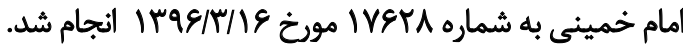

$$
\text { حامي مالب }
$$

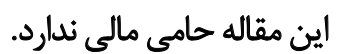

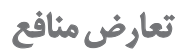

بنا بر اظهارنظر نويسندكان، تعارض منافعى وجود ندارد.

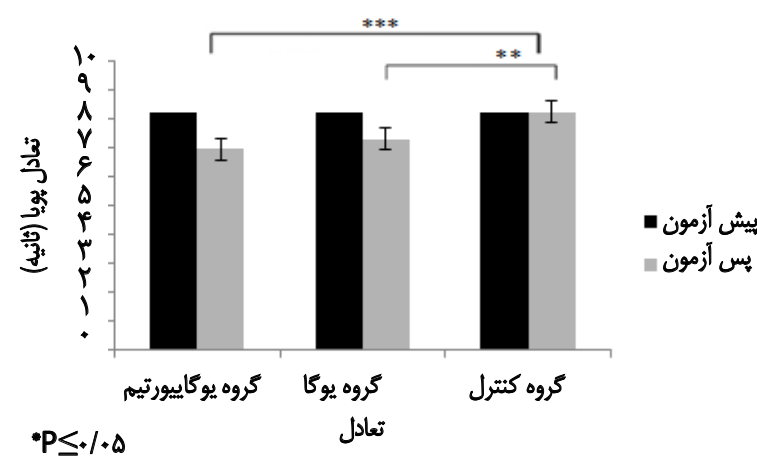

L

تصوير و. مقايسه تعادل يوياى زنان سالمئد بيش و بس از مداخله تحقيقى

حركتى مى تواند تعادل بهترى را براى سالمند رقم بزند. برداشتى كه از اين نتايج حاصل مى إشود اين است كه كاركردهاي

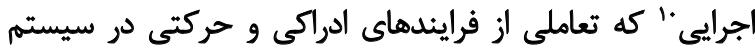

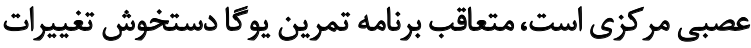

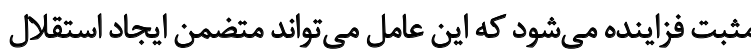

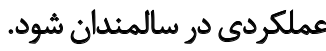

نكثه قابل تأمل در يافتهاي اين يُّوهش، تفاوت نداشتن هيجيك

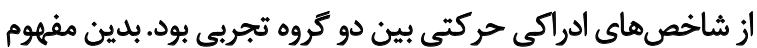

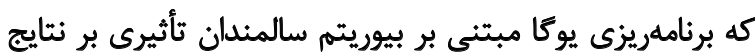

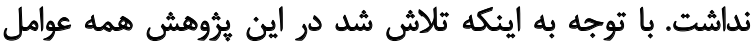

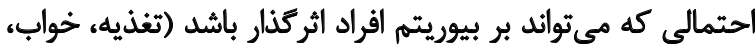

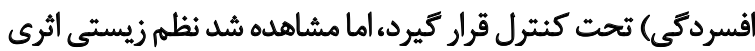

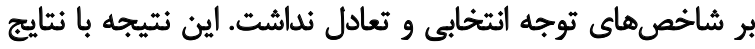

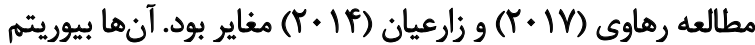

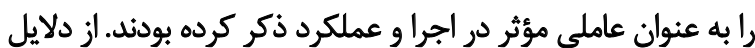

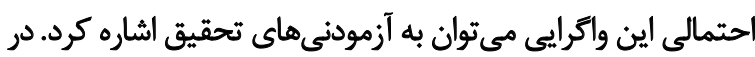

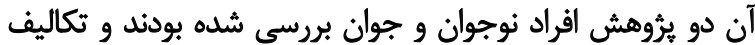

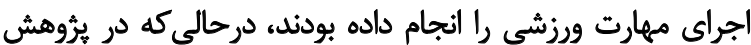

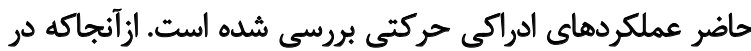

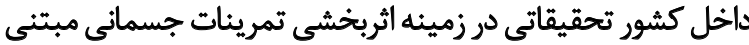

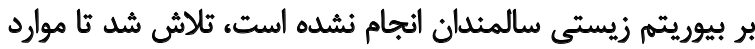
مرتبطتر در مقايسه با نتايج تحقيق حاضر استفادي ماده شود. نتايج اين تحقيق با يافتههاي كيم" و همكاران همخوانى دارد

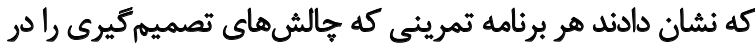

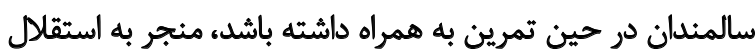

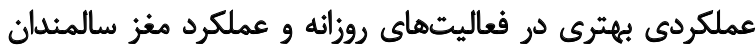

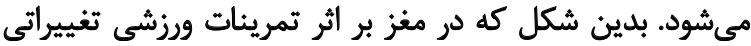

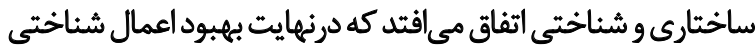




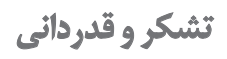

ضمن تشكر از همه افرادى كه در اين يُروهش مشاركت داشتند،

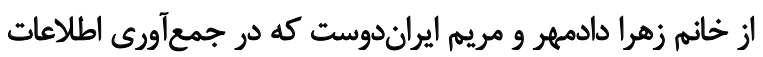

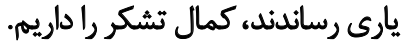




\section{References}

[1] Zareian E, Rabbani V, Saeedi F. The effect of physical biorhythm cycle on some physical fitness factors of adolescent volleyball players. Annals of Applied Sport Science. 2014; 2(1):11-20. [DOI:10.18869/acadpub.aassjournal.2.1.11]

[2] Benca R, Duncan MJ, Frank E, McClung C, Nelson RJ, Vicentic A. Biological rhythms, higher brain function, and behavior: Gaps, opportunities and challenges. Brain Research Reviews. 2009; 62(1):57-70. [DOI:10.1016/j.brainresrev.2009.09.005] [PMID] [PMCID]

[3] Adamantidis A, de Lecea L. Sleep and metabolism: Shared circuits, new connections. Trends in Endocrinology \& Metabolism. 2008; 19(10):362-70. [DOI:10.1016/j.tem.2008.08.007] [PMID]

[4] Ma Y, Olendzki BC, Li W, Hafner AR, Chiriboga D, Hebert JR, et al. Seasonal variation in food intake, physical activity, and body weight in a pre-dominantly overweight population. European Journal of Clinical Nutrition. 2006; 60(4):519-28. [DOI:10.1038/ sj.ejcn.1602346] [PMID] [PMCID]

[5] Kanikowska D, Sato M, Witowski J. Contribution of daily and seasonal biorhythms to obesity in humans. International Journal of Biometeorology. 2015; 59(4):377-84. [DOI:10.1007/s00484-0140871-z] [PMID] [PMCID]

[6] Seidler RD, Bernard JA, Burutolu TB, Fling BW, Gordon MT, Gwin JT, et al. Motor control and aging: Links to age-related brain structural, functional, and biochemical effects. Neuroscience and Biobehavioral Reviews. 2010; 34(5):721-33. [DOI:10.1016/j.neubiorev.2009.10.005] [PMID] [PMCID]

[7] Graveson J, Bauermeister S, McKeown D, Bunce D. Intraindividual reaction time variability, falls, and gait in old age: A systematic review. The Journals of Gerontology Series B, Psychological Sciences and Social Sciences. 2016; 71(5):857-64. [DOI:10.1093/ geronb/gbv027] [PMID]

[8] Sato D, Seko C, Hashitomi T, Sengoku Y, Nomura T. Differential effects of water-based exercise on the cognitive function in independent elderly adults. Aging Clinical and Experimental Research. 2015; 27 (2):149-59. [DOI:10.1007/s40520-014-0252-9] [PMID]

[9] Carral JM, Curras DM, Pérez CA, Suárez MH. Effects of two programmes of combined Land-Based and Water-Based exercise on the cognitive function and fitness levels of healthy older adults. Motriz: Revista de Educação Física. 2017; 23(2). [DOI: 10.1590/ s1980-6574201700020011]

[10] Dagtekin O, Gerbershagen HJ, Wagner W, Petzke F, Radbruch L, Sabatowski R. Assessing cognitive and psychomotor performance under long-term treatment with transdermal buprenorphine in chronic noncancer pain patients. Anesthesia \& Analgesia. 2007; 105(5):1442-8. [DOI:10.1213/01.ane.0000281078.65585.1e] [PMID]

[11] Sadeghi H, Norouzi H, Karimi Asl A, Montazer M. [Functional training program effect on static and dynamic balance in male able-bodied elderly (Persian)]. Iranian Journal of Ageing. 2008; 3(2):565-71.

[12] Lopopolo RB, Greco M, Sullivan D, Craik RL, Mangione KK. Effect of therapeutic exercise on gait speed in community-dwelling elderly people: A meta-analysis. Physical Therapy. 2006; 86(4):520-40. [PMID] [PMID]

[13] Buchner DM, Cress ME, de Lateur BJ, Esselman PC, Margherita AJ, Price R, et al. The effect of strength and endurance training on gait, balance, fall risk, and health services use in community-living older adults. The Journals of Gerontology Series A, Biological Sciences and Medical Sciences. 1997; 52(4):M218-24. [DOI:10.1093/ gerona/52A.4.M218] [PMID]

[14] Irandoust K, Taheri M. [The impact of yoga and pilates exercises on older adults (Persian)]. Iranian Journal of Ageing. 2016; 11(1):152-61. [DOI:10.21859/sija-1101152] [DOI:10.21859/sija1101152]

[15] Kim BK, Rhim YT, Park IS. Effect of regular aerobic exercise on the prevention of cognitive decline and brain disease in elderly people. Journal of Korean Society for the Study of Physical Education. 2013; 18(2):217-29. 
